\title{
LETTERS
}

\section{The Impact of Cost Displays on Ordering}

\author{
Benjamin T. Galen, MD
}

Albert Einstein College of Medicine, Montefiore Medical Center, Division of Hospital Medicine, Eastchester Road, Bronx, NY, USA.

J Gen Intern Med 29(10):1331

DOI: $10.1007 / \mathrm{s} 11606-014-2944-4$

(c) Society of General Internal Medicine 2014

$\mathrm{T}$ o the Editor: Horn et al. demonstrate that a price tag affixed to a diagnostic test can influence physician behavior. ${ }^{1}$ This is not surprising, since physicians are, like anyone else, aware of the value of a dollar. The Medicare reimbursement rate for a given test was a reasonable choice for this price tag, since this is the "cost" (or "charge") to the payer. But does awareness of charge (cost-consciousness) translate into the best care for patients? I am concerned that using price tags to change clinical practice is coercive rather than being value-conscious.

As physicians, our effort to eliminate waste should be focused on using evidence-based diagnostic testing strategies that provide high-yield clinical data at a cost that patients and payers are willing to accept. One example from the inpatient setting of value-based decision-making is the removal of CK-MB from cardiac biomarker panels, since it adds limited clinical value over troponin. ${ }^{2}$

Price tags are coercive because physicians might interpret the cost as they would a personal out-of-pocket expense, rather than in relation to a patient or system's annual healthcare expenditure. Another recent trial showed that a weekly flyer announcing "cost" to residents reduced lab utilization. ${ }^{3}$ Do residents perceive price tags differently than attending physicians? What if your patient is wealthy and self-pays? How does this influence the provider's perception of an assay's value or yield? Too many clinically unimportant factors render price tags on laboratory tests an arbitrary means of rationing.
The majority of tests with price tags in the study by Horn et al. (over $80 \%$ in both the high and low cost categories) were not reduced in utilization by the intervention. The authors do not speculate why some tests were affected and others were not. For example, if a patient and doctor in their study agreed to screen for prostate cancer using prostatespecific antigen (PSA) (one test that was not affected by the intervention) despite the controversial evidence that supports not doing this, did the issue of price even enter into their examination room decision? Certain healthcare networks or payers might someday remove PSA from outpatient screening based on the best available evidence, but this would be a rational decision, not individual rationing by price tag. Reducing laboratory utilization might have a role in cost-control, but let's not confuse the ability to reduce test ordering for evidence that it is the right thing to do.

Corresponding Author: Benjamin T. Galen, MD; Albert Einstein College of Medicine, Montefiore Medical Center, Division of Hospital Medicine, Suite 2-76, 1825, Eastchester Road, Bronx, NY 10461, USA (e-mail: bgalen@montefiore.org).

\section{REFERENCES}

1. Horn DM, Koplan KE, Senese MD, Jrav J, Sequist TD. The Impact of Cost Displays in Primary Care Physician Laboratory Test Ordering. J Gen Intern Med. 2014;29(5):708-14.

2. Saenger A, Jaffe A. Requiem for a heavyweight: the demise of CreatineKinase-MB. Circulation. 2008;118:2200-2206.

3. Stuebing EA, Miner TJ. Surgical Vampires and rising health care expenditure: reducing the cost of daily phlebotomy. Arch Surg. 2011 May; 146(5):524-7. 